\title{
Observação da Interação Professor-Aluno no Jardim de Infância: O Teacher-Pupil Observation Tool
}

\author{
Sofia Major ${ }^{1}$ \\ Universidade dos Açores, Ponta Delgada, Portugal \\ Maria João Seabra-Santos, Maria Filomena Gaspar, Mariana Pimentel, \\ Andreia F. Azevedo, Tatiana C. Homem, Elsa Baptista \\ Universidade de Coimbra, Coimbra, Portugal
}

\section{RESUMO}

Este estudo teve como objetivo apresentar a versão portuguesa do Teacher-Pupil Observation Tool (T-POT), no que diz respeito ao acordo e fidedignidade entre observadores e às categorias de comportamentos observadas nos professores na sua utilização em contexto pré-escolar. Três observadores receberam treinamento na cotação de 11 categorias de comportamentos do professor do T-POT. Posteriormente, 65 professoras de jardim de infância foram observadas durante 25 minutos, numa situação de interação nas suas salas com um pequeno grupo de crianças. Obteve-se uma média de acordo entre observadores de $74 \%$ nas sessões de treinamento e coeficientes de correlação intraclasse de $0,90-0,99$. Os resultados indicam que as categorias do T-POT mais utilizadas pelas professoras nas suas interações com as crianças são questões, confirmações e ordens. A discussão salienta as implicações para a prática e a pesquisa da utilização de medidas de observação na avaliação da interação professor-aluno nas salas de jardim de infância. Palavras-chave: observação; interação professor-aluno; pré-escolares; fidedignidade.

\section{ABSTRACT - Observation of teacher-student interaction in preschool: The Teacher-Pupil Observation Tool}

The purpose of this study was to present the Portuguese version of the Teacher-Pupil Observation Tool (T-POT), in terms of agreement and reliability among observers and the categories of behaviors observed in teachers when used in preschool setting. Three observers were trained in scoring 11 T-POT teacher's behavior categories. Subsequently, 65 preschool teachers were observed with T-POT during 25 minutes, while interacting in their classrooms with a small group of children. A mean of $74 \%$ of agreement was reached between observers for the training sessions and intraclass correlation coefficients of $0,90-0,99$. The results indicate that the T-POT categories most used by teachers in their interactions with children are questions, acknowledgments and commands. The discussion highlights the implications for practice and research on the use of observation measures in the assessment of teacherstudent interaction in preschool classrooms.

Keywords: observation; teacher-student interaction; preschool children; reliability.

RESUMEN - Observación de la interacción profesor-alumno en la educación infantil: El Teacher-Pupil Observation Tool Este estudio tuvo como objetivo presentar la versión portuguesa del Teacher-Pupil Observation Tool (T-POT), en lo que se refiere al acuerdo y la confiabilidad entre observadores y, por otro lado, las categorías de comportamiento observadas en los profesores en la utilización del test en el contexto preescolar. Tres observadores recibieron entrenamiento en la cotización de 11 categorías de comportamiento del profesor del T-POT. Posteriormente, 65 profesoras de la educación infantil fueron observadas utilizando el T-POT durante 25 minutos, en una situación de interacción en sus aulas con un pequeño grupo de niños. Se obtuvo un acuerdo medio del $74 \%$ entre observadores en las sesiones de entrenamiento y coeficientes de correlación intraclase de 0,90-0,99. Los resultados indican que las categorías del T-POT más utilizadas por las profesoras en sus interacciones con los niños son cuestiones, confirmaciones y órdenes. La discusión destaca las implicaciones para la práctica y la investigación de la utilización de medidas de observación en la evaluación de la interacción profesoralumno en la educación infantil.

Palabras clave: observación; interacción profesor-alumno; preescolares; confiabilidad.

Nas últimas três décadas tem-se assistido a uma mudança na relação professor-aluno, com as múltiplas dinâmicas das salas de aula a revelarem-se progressivamente mais desafiantes para os professores (Scott, Alder, \& Hirn, 2011), que referem maiores dificuldades na gestão dos problemas de comportamento dos alunos (Thangarajathi

${ }^{1}$ Endereço para correspondência: Faculdade de Ciências Sociais e Humanas, Universidade dos Açores, Campus de Ponta Delgada. Rua da Mãe de Deus, $9500-321$ Ponta Delgada, Portugal. Tel.: +351 29665-0000. E-mail: sofia.o.major@uac.pt

O presente artigo insere-se no projeto "Anos Incríveis para a Promoção da Saúde Mental" apoiado pelo Programa Iniciativas em Saúde Pública (PT06), financiado pelo EEA Grants Financial Mechanism 2009-2014 (51SM04). 
\& Enok, 2010; Vujnovic et al., 2014). Nesse contexto, a literatura tem evidenciado que crianças em idade pré-escolar com problemas de comportamento se encontram numa posição de risco acrescido desses problemas precoces se desenvolverem em perturbação de conduta, bem como num menor ajustamento social e emocional, insucesso/abandono escolar, e delinquência (WebsterStratton, Reid, \& Stoolmiller, 2008). Em contrapartida, as relações professor-aluno têm sido reconhecidas como desempenhando um papel essencial no desenvolvimento social e emocional das crianças, com impacto no seu futuro sucesso escolar (Hutchings et al., 2007; Pianta \& Stuhlman, 2004; Thangarajathi \& Enok, 2010; Vujnovic et al., 2014).

Dado que os professores desempenham um papel central no desenvolvimento das crianças em idade pré-escolar (Hutchings et al., 2007; Strickland, Hopkins, \& Keenan, 2012), que vai muito para além da instrução (Scott et al., 2011), o ambiente da sala é um elemento-chave para a aprendizagem e ensino (Cadima \& Leal, 2008; Thangarajathi \& Enok, 2010). Acresce que existe evidência de que os problemas de comportamento identificados em contexto escolar não se limitam à criança que apresenta esses problemas, mas acabam por afetar, igualmente, os pares e os professores (Carlson, Tiret, Bender, \& Benson, 2011). Se os problemas de comportamento das crianças em sala de jardim de infância têm um impacto negativo na relação professor-aluno (Hutchings et al., 2007), também alguns comportamentos dos professores (e.g., utilização de estratégias disciplinares negativas) têm sido associados a comportamentos negativos das crianças na sala e a um desempenho acadêmico mais fraco. No sentido oposto, um ambiente de sala acolhedor e positivo, nomeadamente por meio da utilização de estratégias positivas (e.g., utilização de elogios específicos perante a ocorrência de comportamentos apropriados) (Webster-Stratton et al., 2008), melhora o desempenho acadêmico e encontra-se associado a um melhor ajustamento social, emocional e comportamental por parte das crianças (Hutchings, Martin-Forbes, Daley, \& Williams, 2013; WebsterStratton et al., 2008). É nesse sentido que Pianta e Stuhlman (2004) consideram a relação professor-aluno como um elemento-chave para o ajustamento das crianças na escola, bem como um indicador desse mesmo ajustamento. No entanto, apesar do crescente interesse por essa temática, a observação das interações professor-aluno carece de mais pesquisa. Um indicador dessa realidade é a revisão de literatura realizada no Brasil, na qual foi analisada a produção científica de 1970 a 2006 com recurso ao método observacional, em que Cano e Sampaio (2007) verificaram que, das 116 pesquisas consideradas, os temas mais comuns se referiam, nomeadamente, a crianças em diversas situações $(23,3 \%)$, à utilização da observação na formação de psicólogos (10,4\%), técnicas e conceitos associados à observação (8,6\%), sendo que 19,8\% se referiam à relação mãe-bebê e apenas $5,2 \%$ à relação professor-aluno.

Diversos métodos têm sido utilizados para avaliar o que se passa na sala de aula, tais como medidas indiretas (e.g., entrevista) e diretas (e.g., observação direta) (Scott et al., 2011). Nesse contexto, a observação é apontada como uma das medidas mais diretas e objetivas e pode ser utilizada para várias finalidades: despistagem, avaliação de diagnóstico, formulação e monitorização de estratégias de intervenção, e investigação (Lewis, Scott, Wehby, \& Howard, 2014; Merrell, 2008; Volpe, DiPerna, Hintze, \& Shapiro, 2005). As principais vantagens da utilização da observação em contexto escolar são o fato de esta permitir a observação em meio natural e a coleta de dados "ao vivo". Cohen, Manion e Morrison (2007) destacam ainda outras vantagens: (a) ultrapassar problemas relacionados com o intervalo de tempo entre o comportamento observado e o registro do acontecimento, (b) ir além das perceções dos respondentes recolhidas por meio de outros métodos de avaliação, e (c) trazer alguma "frescura" na coleta de dados (os dados obtidos por meio da observação são menos previsíveis do que numa avaliação com outros métodos). Acresce que, em termos de procedimento de coleta de dados, os professores podem preferir ser avaliados na presença de um observador em sua sala em vez de terem de preencher inventários extensos fora do seu horário laboral (Cohen, Manion, \& Morrison 2007). Apesar da sua utilidade, a validade da observação direta pode ser comprometida por diversos fatores, tais como reduzido acordo interobservadores, definição pobre das categorias de comportamentos a observar, reatividade do observado, bias do observador e especificidade situacional do comportamento alvo (Merrell, 2008). A essas dificuldades podem acrescentar-se outras, como não captar todas as variáveis contextuais (e.g., número de alunos, momento do dia), o impacto do comportamento das crianças na sala e a complexidade de variáveis sequenciais, uma vez que, na sala, podem ocorrer diversos acontecimentos simultâneos, interativos e até multiplicativos (Lewis et al., 2014). No entanto, a principal limitação da observação é ser um procedimento moroso e dispendioso, que requer um longo período de treinamento para garantir a obtenção de níveis satisfatórios de fidedignidade entre observadores (Alvarenga \& Cerezo, 2014; Cohen et al., 2007). Ainda assim, Volpe, et al. (2005) salientam que grande parte dessas limitações podem ser minimizadas com recurso a instrumentos de avaliação devidamente validados e a um adequado treinamento dos observadores.

Encontram-se disponíveis diversas ferramentas de observação para se utilizar em salas de jardim de infância (Vujnovic et al., 2014), com algumas a assumirem a forma de escalas de avaliação em que o avaliador tem 
de fazer inferências acerca do comportamento observado (Cohen et al., 2007). Dentro dessa primeira categoria, encontra-se o Classroom Practices Inventory (CPI; Hyson, Hirsh-Pasek, \& Rescorla, 1990), composto por 26 itens que avaliam a implementação de práticas desenvolvimentais adequadas. O CPI está organizado em duas subescalas, Clima Emocional (entusiasmo do professor, encorajamento e orientação positiva) e Foco no Programa (características do currículo), em que cada item é cotado numa escala de cinco pontos. Por sua vez, o Assessment Profile for Early Childhood Programs (AbbottShim, Lambert, \& McCarty, 2000) é uma checklist de observação com 75 itens organizados em cinco subscalas (e.g., Horário, Currículo). Cada item é cotado segundo uma escala dicotômica (sim/não) em referência a um período de observação de 15-20 minutos por hora, conduzido ao longo de três horas. Existe ainda a Early Childhood Environment Rating Scale - Revised (ECERS-R; Harms, Clifford, \& Cryer, 1998), com 43 itens (cotados numa escala de sete pontos), sendo utilizada na avaliação de programas de intervenção precoce. Requer cerca de três horas de observação e avalia a qualidade do ambiente da sala por meio de sete escalas (e.g., Espaço e Mobiliário), com apenas uma escala (i.e., Interação) focada na relação aluno-professor. A ECERS-R está disponível em Portugal (Escala de Avaliação do Ambiente em Educação de Infância - Edição Revista; Abreu-Lima \& Nunes, 2006), sendo utilizada pelo Ministério da Educação para a monitorização e acompanhamento do desenvolvimento curricular na educação pré-escolar.

Apesar das boas propriedades psicométricas associadas a essas escalas de avaliação (Abbott-Shim et al., 2000; Harms et al., 1998), essas ferramentas requerem longos períodos de observação e estão demasiado centradas numa avaliação da qualidade das condições da sala e das práticas dos professores, e não tanto na interação professor-aluno. Alguns instrumentos procuraram ultrapassar essa última limitação. É o caso do Classroom Assessment Scoring System (CLASS; Pianta, LaParo, \& Hamre, 2008), quando complementado com o Individualized Classroom Assessment Scoring System (inCLASS; Downer, Booren, Lima, Luchner, \& Pianta, 2010), que permite a avaliação das interações sociais entre uma criança-alvo e o seu professor. As interações são operacionalizadas em 10 dimensões, cotadas de 1 a 7 , que se encontram agrupadas em três domínios (Apoio Emocional, Organização da Sala e Apoio a Nível de Instrução). A observação é realizada ao longo de vários períodos de 20 minutos. No entanto, mantém-se a limitação na captura da complexidade das interações professor-aluno, uma vez que não permitem a quantificação objetiva das respostas do professor (Vujnovic et al., 2014). Cadima e Leal (2008) procederam à tradução para português do CLASS (Sistema de Observação das Interações na Sala de Aula), para ser utilizado no $1^{\text {o }}$ ciclo de escolaridade básica. Já Vujnovic et al. (2014) apresentaram um estudo preliminar da adaptação para o pré-escolar do Student Behavior Teacher Response (SBTR; Pelham, Greiner, \& Gnagy, 2008), focado na avaliação do comportamento do professor perante um comportamento desafiador (quebra de uma regra da sala) do aluno. A observação tem a duração de 30 minutos e o comportamento do professor é cotado quanto à confirmação do comportamento desafiador (por parte do professor) e consequência do comportamento do aluno. Para tentar contornar a morosidade e custos financeiros necessários para utilizar procedimentos de observação em sala de aula, Martin et al. (2010) propuseram um sistema de codificação de registro simples: Teacher-Pupil Observation Tool (T-POT). O T-POT foi desenvolvido para ultrapassar algumas das limitações das medidas de observação em sala existentes, procurando utilizar componentes do Dyadic Parent-Child Interaction Coding System (DPICS; Robinson \& Eyberg, 1981).

Vujnovic et al. (2014) salientam que a compreensão da resposta dos professores ao comportamento das crianças contribui para interações professor-aluno mais efetivas, sendo o desenvolvimento de ferramentas de avaliação tecnicamente adequadas essencial. Nesse sentido, este estudo pretendeu, num primeiro momento, apresentar evidências de fidedignidade entre observadores quando utilizam a versão portuguesa do T-POT. Num segundo momento, recorreu-se ao T-POT em contexto de jardim de infância para identificar as estratégias mais e menos utilizadas por professores nas suas salas.

\section{Método}

\section{Participantes}

A amostra utilizada no presente estudo foi recolhida no âmbito do projeto "Anos Incríveis para a Promoção da Saúde Mental" (EEA Grants - 51SM4), desenvolvido na Faculdade de Psicologia e de Ciências da Educação, Universidade de Coimbra (Portugal). Sessenta e cinco professores de 53 jardins de infância públicos aceitaram participar do estudo de eficácia de uma intervenção em sala de jardim de infância. As professoras (todas do gênero feminino) tinham entre 42-61 anos $(M=52,53$; $D P=3,65)$. Em termos de formação acadêmica, 57 $(87,7 \%)$ tinham um bacharelado e oito (12.3\%) tinham completado uma licenciatura. Todas as professoras tinham uma considerável experiência prévia: entre 12-39 anos $(M=28,92, D P=4,36)$. Nas suas salas, trabalhavam com 10 a 25 crianças $(M=17,34 ; D P=4,18)$.

\section{Instrumentos}

O questionário sociodemográfico (professores) foi desenvolvido no âmbito do projeto e permitiu a coleta de dados de identificação do professor (e.g., idade, estado civil), formação (e.g., tipo de formação), percurso profissional (e.g., número de anos de serviço) e informação 
referente ao respetivo grupo de crianças (e.g., número de meninos e meninas).

O Teacher-Pupil Observation Tool (T-POT) é uma medida de observação direta que envolve a contagem de frequência de comportamentos de professores e crianças. Consiste em 75 categorias de itens, com cerca de metade referentes ao comportamento do professor (e.g., comportamento positivo, elogio específico), e metade em referência ao comportamento da criança (e.g., obediência, comportamento off-task) (Martin et al., 2010; Versão portuguesa: Gaspar, Leitão, \& Vale, 2011). No estudo de desenvolvimento do T-POT, as 75 categorias de itens foram posteriormente agrupadas em oito categorias compósitas para análise dos resultados (duas em relação ao professor e seis em relação às crianças) (Martin et al., 2010). Os observadores alcançaram um nível de acordo de $70 \%$ ou superior com cerca de 23 horas de treino. O cálculo dos coeficientes de correlação intraclasse indicou uma boa estabilidade intercotadores com um valor médio de 0,78 para as 75 categorias de comportamento (amplitude de 0,59 a 0,99 ).
A evidência de validade discriminante foi demonstrada por correlações positivas entre as categorias de comportamentos negativos dos professores e problemas sociais, emocionais e de comportamento das crianças; e entre as categorias de comportamentos positivos dos professores e ajustamento social e comportamental das crianças (Martin et al., 2010). O T-POT foi utilizado em diversos estudos na avaliação da eficácia de um programa de intervenção em sala de jardim de infância (Incredible Years - Teacher Classroom Management Program; WebsterStratton, 2003), no País de Gales (Hutchings et al., 2007; Hutching et al., 2013) e na Irlanda (McGilloway et al., 2010), com médias de acordo entre observadores a rondarem os $70 \%$. Tendo em conta os objetivos do projeto no qual este estudo se insere, foram utilizadas apenas 11 categorias de itens referentes a comportamentos do professor: Confirmação, Comportamento Negativo, Elogio Não Específico, Elogio Específico, Comportamento Positivo, Resolução de Problemas, Ignorar, Questões, Ordens Indiretas, Ordens Diretas e Aviso de Tempo de Pausa (cf. Tabela 1).

Tabela 1

Comportamentos Incluídos nas 11 Categorias do T-POT

\begin{tabular}{|c|c|c|}
\hline Categoria & Comportamento & Exemplo \\
\hline \multirow[t]{3}{*}{ Confirmação } & Confirmação & Está bem. \\
\hline & Comentário/questão reflexiva & $\begin{array}{l}\text { Criança: Vaca muu. } \\
\text { Professor: A vaca faz muu }\end{array}$ \\
\hline & Comentário/questão descritiva & Estás a contar uma história. \\
\hline \multirow{6}{*}{$\begin{array}{l}\text { Comportamento } \\
\text { Negativo }\end{array}$} & Criticismo & És mau. \\
\hline & Ordem negativa & Para já com isso. \\
\hline & Comportamento físico negativo & $\begin{array}{l}\text { Professor agarra a criança para } \\
\text { a impedir de fazer algo. }\end{array}$ \\
\hline & Intrusão & $\begin{array}{l}\text { Professor afasta algo da criança } \\
\text { quando ela o está a usar. }\end{array}$ \\
\hline & Avisos & Se não fizeres... não vais... \\
\hline & Sons & “Shtt” \\
\hline Elogio Não-Específico & Elogio não-específico & Boa. \\
\hline Elogio Específico & Elogio específico & Fizeste um trabalho de pintura excelente. \\
\hline \multirow{4}{*}{$\begin{array}{l}\text { Comportamento } \\
\text { Positivo }\end{array}$} & Afeto positivo & Sorriso. \\
\hline & Afeto físico positivo & Abraço. \\
\hline & “Quando...então" & Depois de acabares o desenho podes ir brincar. \\
\hline & Encorajamento & Huau! \\
\hline $\begin{array}{l}\text { Resolução de } \\
\text { Problemas }\end{array}$ & Resolução de problemas & Pensa numa maneira de... \\
\hline Ignorar & Ignorar & $\begin{array}{l}\text { Criança: Choraminga lamuriando-se. } \\
\text { Professor: Não emite qualquer comentário } \\
\text { verbal ou resposta física. }\end{array}$ \\
\hline Questões & Questões & Quantas flores estão aqui? \\
\hline Ordens Indiretas & Ordens indiretas & Põe isso aqui, está bem? \\
\hline Ordens Diretas & Ordens diretas & Vem aqui ao pé de mim, por favor. \\
\hline Aviso Tempo Pausa & Aviso tempo pausa & $\begin{array}{l}\text { Se continuas a comportar-te } \\
\text { assim vais para a pausa. }\end{array}$ \\
\hline
\end{tabular}




\section{Treinamento dos Observadores}

A observação foi realizada por três psicólogas que passaram por um treinamento prévio que envolveu várias fases: (a) análise do manual (familiarização com as categorias do T-POT e exemplos de comportamentos abrangidos por cada uma das 11 categorias; (b) observação e codificação de 28 cenas de DVDs, referentes a interações professor-aluno (consolidação no treinamento); e (c) observação e codificação "ao vivo" em salas de jardim de infância (treinamento de codificação em contexto real). As fases B e C envolveram comparação/discussão do número de comportamentos observados, com especial atenção para os casos de desacordo (isto é, situações em que uma observadora cotava um comportamento e outra não). Foram realizadas sete sessões de treinamento na fase $\mathrm{B}$, perfazendo um total de 21 horas de codificação/discussão de cenas de DVDs.

\section{Coleta de Dados}

A avaliação foi efetuada no início do ano letivo (outubro-novembro), antes da implementação do programa de intervenção (linha de base). As 65 salas foram repartidas pelas três observadoras com a seguinte distribuição: 26, 24 e 15 salas. Todos os momentos de observação foram previamente agendados com as professoras e tiveram lugar nos respetivos jardins de infância. As observações foram realizadas no período da manhã, após a receção das crianças pelas professoras e cumprimento das rotinas da manhã (e.g., canção dos bons dias, marcação de presenças), ou no período da tarde (logo após a hora de almoço). Em cada uma das 65 salas envolvidas no estudo, as observações foram realizadas com um grupo de crianças (entre quatro e seis crianças por grupo observado) selecionado pelas professoras, sem indicação de qualquer critério acerca das crianças a incluir no grupo. Quanto à atividade, as professoras recebiam como instrução a escolha de uma atividade de mesa em pequeno grupo, procurando que esta fosse o mais próxima possível da sua rotina diária. As professoras eram também instruídas no sentido de atuar da forma o mais natural possível, ignorando a presença da observadora, que iria adotar uma atitude não intrusiva para não afetar a dinâmica da atividade. A observação teve a duração de 25 minutos, subdividida em períodos de 5 minutos cada, em cada uma das 65 salas. Por meio da grelha do T-POT, cada vez que um comportamento era observado, um sinal era marcado na respetiva categoria.

De forma a salvaguardar os aspectos éticos, o projeto mais vasto onde este estudo se inseriu obteve autorização da Comissão Nacional de Proteção de Dados (Comitê de Ética em Pesquisa de Portugal). Uma vez obtidas as permissões das escolas, foram realizadas reuniões com as professoras sendo-lhes apresentados os objetivos do projeto e solicitada a assinatura de um documento de consentimento informado. As observações em sala foram, igualmente, precedidas da assinatura de um documento de consentimento informado por parte dos pais/responsáveis pelas crianças.

\section{Análise dos Dados}

Todas as análises estatísticas foram realizadas com a Versão 24 do software estatístico IBM SPSS Statistics. Seguindo as indicações do manual e as análises efetuadas com a versão original do T-POT (Martin et al., 2010), num primeiro momento foi calculada a percentagem de acordo entre observadores para as 28 cenas de DVD cotadas nas sessões de treinamento, por meio da fórmula (Acordo/Acordo+Desacordo) ${ }^{\star} 100$, assim como o coeficiente de correlação intraclasse (CCI). Num segundo momento, as estatísticas descritivas (mínimo-máximo, médias, desvios padrão) foram utilizadas para analisar os resultados obtidos para as 11 categorias do T-POT quando da observação das interações professor-aluno.

\section{Resultados}

\section{Acordo e Fidedignidade entre Observadores}

No decorrer das sessões de treinamento na cotação do T-POT, as três observadoras alcançaram um nível médio de acordo de $74 \%$ na codificação das 28 cenas. As categorias Resolução de Problemas, Ignorar e Tempo de Pausa apresentaram uma frequência nula e, como tal, não foram consideradas no cálculo do acordo. As percentagens médias do acordo entre as três observadoras oscilaram entre 67 (Comportamento Positivo) e 78\% (Questões e Ordens Diretas) (cf. Tabela 2).

Posteriormente foram calculados os CCIs para as mesmas categorias. Segundo os dados incluídos na Tabela 2, os coeficientes de correlação oscilaram entre 0,90 (Comportamento Positivo) e 0,99 (Confirmação).

\section{Estatísticas Descritivas}

A Tabela 3 apresenta as estatísticas descritivas para as 11 categorias do T-POT, quando este foi utilizado para observar o comportamento das professoras. As categorias do T-POT mais observadas foram as Questões $(M=82,48, D P=30,46)$, Confirmação $(M=52,11, \quad D P=16,94) \quad$ e Ordens (indiretas) $(M=47,14, D P=24,82)$. As categorias de comportamento menos observadas referem-se ao Aviso Tempo de Pausa $(M=0,03, D P=0,17)$, Resolução de Problemas $(M=1,08, D P=2,14)$ e Elogio Específico $(M=1,91$, $D P=2,77)$. Por outro lado, observou-se uma grande variabilidade de comportamentos entre as salas/professoras, podendo um comportamento não ser observado nenhuma vez numa sala e sê-lo 65 vezes em outra (como é o caso do Elogio Não Específico), ou 22 vezes numa e 165 em outra (como acontece com as Questões) (cf. Tabela 3). A categoria Ignorar não foi observada em qualquer uma das 65 salas. 
Tabela 2

Média de Acordo entre Observadores e Coeficiente de Correlação Intraclasse (CCI) com Intervalo de Confiança: Categorias T-POT nas Sessões de Treinamento

\begin{tabular}{lccc}
\hline \multicolumn{1}{c}{ Categoria T-POT } & Média Acordo (\%) & CCI & Intervalo Confiança 95\% \\
\hline Confirmação & 77 & 0,99 & $0,98-0,99$ \\
Comportamento Negativo & 74 & 0,93 & $0,85-0,97$ \\
Elogio Não Específico & 69 & 0,94 & $0,89-0,97$ \\
Elogio Específico & 73 & 0,93 & $0,87-0,97$ \\
Comportamento Positivo & 67 & 0,90 & $0,79-0,95$ \\
Resolução Problemasa $^{a}$ & - & - & - \\
Ignorar $^{\text {a }}$ & - & - & - \\
Questões $_{\text {Ordens Indiretas }}$ & 78 & 0,94 & $0,89-0,97$ \\
Ordens Diretas $_{\text {Aviso Tempo Pausa }}{ }^{a}$ & 74 & 0,95 & $0,89-0,98$ \\
\hline
\end{tabular}

Nota. ${ }^{\text {a }}$ Categorias de comportamento com frequência nula nas 28 cenas observadas

Tabela 3

Estatísticas Descritivas: Categorias T-POT na Observação nas 65 Salas

\begin{tabular}{lccc}
\hline \multicolumn{1}{c}{ Categoria T-POT } & Mín-Max & M & DP \\
\hline Confirmação & $18-101$ & 52,11 & 16,94 \\
Comportamento Negativo & $1-87$ & 30,94 & 21,80 \\
Elogio Não Específico & $0-65$ & 16,11 & 12,69 \\
Elogio Específico & $0-13$ & 1,91 & 2,77 \\
Comportamento Positivo & $1-45$ & 20,55 & 10,75 \\
Resolução Problemas & $0-11$ & 1,08 & 2,14 \\
Ignorar & - & - & - \\
Questões & $22-165$ & 82,48 & 30,46 \\
Ordens Indiretas & $3-128$ & 47,14 & 24,82 \\
Ordens Diretas & $6-89$ & 39,77 & 21,02 \\
Aviso Tempo Pausa & $0-1$ & 0,03 & 0,17 \\
\hline
\end{tabular}

Nota. ${ }^{a}$ Categoria de comportamento com frequência nula (isto é, não observado)

\section{Discussão}

Atendendo ao papel de relevo da interação professor-aluno no ajustamento social, emocional e comportamental das crianças (Hutchings et al., 2007; Vujnovic et al., 2014), o desenvolvimento de ferramentas de observação em contexto escolar e para faixas etárias mais novas, como os pré-escolares, revela-se premente. O presente estudo teve por objetivo analisar o acordo e a fidedignidade entre observadores após treinamento e as estratégias utilizadas por professores de jardim de infância durante uma interação com um pequeno grupo de crianças, com recurso a categorias do comportamento do professor da versão portuguesa do T-POT. Diversos estudos prévios já demonstraram a eficácia e utilidade desse instrumento de observação enquanto medida da interação professor-aluno (Hutchings et al., 2007; Hutchings et al., 2013; McGilloway et al., 2010).
Ao utilizar a observação na recolha de dados, o treinamento é uma fase crucial uma vez que permite que os vários observadores envolvidos utilizem as mesmas definições operacionais, registrem a mesma observação de forma idêntica e adquiram experiência (Cohen et al., 2007). Ao longo das sessões de treinamento das três observadoras, foi possível alcançar um grau de acordo de $74 \%$, que vai ao encontro da recomendação do manual do T-POT de os observadores estarem aptos a utilizar o T-POT ao alcançarem um grau de acordo de $70 \%$ (e o valor considerado aceitável para o grau de acordo com instrumentos desse tipo). Esse valor é também muito próximo do valor de 70\% referido por Martin et al. (2010) no estudo do desenvolvimento do T-POT e dos $73 \%$ obtidos no estudo de McGilloway et al. (2010). Os valores de acordo ligeiramente abaixo dos $70 \%$ para as categorias de Comportamento Positivo (69\%) e Elogio Não Específico $(67 \%)$ ficam a dever-se a alguma dificuldade inicial na discriminação entre esses dois tipos de comportamentos 
e apontam para a necessidade de maior atenção a essa distinção durante o treinamento dos observadores. Aliás, o manual do T-POT faz referência à dificuldade de diferenciação de comportamentos de encorajamento como "Huau" (incluídos na categoria de Comportamento Positivo) com o elogio não específico, podendo ser considerados como elogios não específicos borderline (Gaspar et al., 2011). Quanto ao CCI, utilizado como medida de fidedignidade entre observadores, todos os coeficientes obtidos se situam acima de 0,90 , demonstrando a proximidade dos comportamentos cotados pelas três observadoras independentes perante as mesmas cenas de DVDs, estando de acordo com os valores recomendados $\geq 0,90$ (Cohen et al., 2007). Em congruência com os dados do acordo entre observadores, o valor mais baixo obtido foi para o Comportamento Positivo (intervalo de confiança a 95\% com maior amplitude: 0,79-0,95).

A utilização da grelha de observação T-POT junto de 65 professoras permitiu identificar o recurso a muitas Questões, Confirmação e Ordens (indiretas). As categorias menos observadas foram o Aviso de Tempo de Pausa, Resolução de Problemas e Elogio Específico. Destaca-se que o ignorar foi uma estratégia que não foi utilizada por nenhuma das professoras. Como indicam as estatísticas descritivas, há professoras que não utilizam estratégias como o elogio ou a resolução de problemas (mínimo de 0 para essas categorias). Esses resultados parecem indicar alguma falta de objetividade e especificidade nas indicações dadas por algumas professoras às crianças (recurso a muitas questões e ordens indiretas durante as interações). Destaca-se ainda a necessidade de dar maior importância aos comportamentos positivos das crianças, recorrendo a mais elogios (nomeadamente elogios específicos), assim como adotar uma postura mais proativa na interação com as crianças, guiando e encorajando a resolução de problemas por parte das crianças.

Diversas potencialidades da presente pesquisa merecem ser salientadas, nomeadamente a dimensão da amostra (65 professoras face a uma amostra de 12 professoras no estudo de desenvolvimento da versão original do T-POT), representando um ponto favorável à generalização dos resultados obtidos. A duração das sessões de treinamento (cerca de três horas cada) também foi um ponto positivo, uma vez que o treinamento dos observadores é uma das melhores estratégias para alcançar bons níveis de acordo. Por outro lado, o recurso a uma ferramenta de observação, como o T-POT, uma forma de registro de evento (ou sistema de sinais), em que é registrado um sinal sempre que um determinado comportamento ocorre, permite obter um retrato mais claro da frequência/incidência dos comportamentos dos professores.

Apesar das vantagens, algumas limitações merecem ser discutidas, tais como terem sido as professoras a selecionar as crianças a incluir no grupo-alvo de observação. Enquanto algumas professoras podem ter escolhido as crianças mais bem-comportadas do grupo para evitarem situações problemáticas durante a observação, outras podem ter optado por incluir as "mais problemáticas" com o objetivo de demonstrar à observadora as dificuldades em lidar com o comportamento dessas crianças da sua sala. Acresce que, na instrução dada às professoras, apenas foi pedido que escolhessem uma atividade de mesa. Essa falta de especificação do tipo de tarefa a desenvolver durante a observação levou a uma não uniformidade nas tarefas observadas, com algumas professoras a optarem por tarefas mais tranquilas ou mais ativas com necessidades distintas de intervenção da professora (e.g., colorir versus tocar instrumentos musicais), o que se pode ter refletido na observação de padrões distintos de interação professora-aluno. Uma indicação mais precisa no Manual do T-POT sobre um possível leque de atividades a desenvolver poderia introduzir um elemento adicional de estandardização no procedimento. Por fim, três das 11 categorias consideradas no estudo não foram observadas nas sessões de treinamento.

Estando o T-POT traduzido para a língua portuguesa, pesquisas futuras em países como o Brasil poderão contribuir para ampliar as evidências de fidedignidade dessa medida, bem como a utilização da versão completa do T-POT, de forma a estudar também as categorias comportamentais da criança. A análise das várias categorias do T-POT atendendo a diversas variáveis, tais como número de alunos por sala, número de anos de experiência dos professores, nível etário e gênero dos alunos do grupo, poderá também trazer novas contribuições à utilização dessa ferramenta de observação. Por fim, pesquisas futuras que procurem analisar a relação do T-POT com instrumentos de autorrelato, e a sua capacidade para identificar modificações que ocorram no comportamento de professores e alunos, após a participação num programa de intervenção, poderão trazer contribuições significativas nos estudos de evidência de validade.

\section{Considerações Finais}

Considerando a importância da observação na avaliação psicológica e a relevância da relação professor-aluno no desenvolvimento (e.g., social, emocional, acadêmico) das crianças, a presente pesquisa procurou analisar a fidedignidade alcançada no treinamento de observadores com o T-POT, assim como na capacidade do instrumento para identificar os comportamentos mais e menos utilizados nas interações professor-aluno em salas de jardim de infância. Se é certo que temos assistido a muitos avanços nessa área, nomeadamente no que diz respeito ao desenvolvimento de instrumentos de avaliação do tipo escala/questionário, a compreensão do comportamento das crianças e professores em contexto de sala de jardim de infância pode ser consideravelmente aprofundada por meio do uso de uma grelha de observação como o T-POT. Nossos achados 
permitem ainda concluir que, dentro das medidas de observação da interação professor-aluno, o T-POT se revela uma medida amigável para as crianças e professores, ao remeter para atividades do dia a dia a que já estão habituados, não sendo muito intrusivo. A utilidade do
T-POT destaca-se também enquanto ferramenta interessante ao ajudar o professor a refletir acerca da sua prática, dando-lhe uma imagem da sua interação com o aluno, que poderá servir de linha de base para futuras intervenções.

\section{Referências}

Abbott-Shim, M., Lambert, R., \& McCarty, F. (2000). Structural model of Head Start classroom quality. Early Childhood Research Quarterly, 15(1), 115-134. doi: 10.1016/S0885-2006(99)00037-X

Abreu-Lima, I., \& Nunes, C. (2006). A Escala de Avaliação do Ambiente em Educação de Infância. Versão Revista (ECERS-R). Atas da XI Conferência Internacional. Avaliação Psicologia: Formas e Contextos (pp. 633-643). Braga: Psiquilibrios.

Alvarenga, P., \& Cerezo, M. A. (2014). Fidedignidade da versão brasileira do Sistema SOCIII para análise da interação mãe-criança. Avaliação Psicológica, 13(1), 47-56.

Cadima, J., \& Leal, T. (2008). Observação dos processos interativos em salas do $1^{\circ}$ ciclo do ensino básico. Em A. P. Noronha, C. Machado, L. S. Almeida, M. Gonçalves, S. Martin, \& V. Ramalho (Eds.). Atas das XIII Conferência Internacional de Avaliação Psicológica: Formas e Contextos. Braga: Psiquilibrios.

Cano, D. S., \& Sampaio, I. T. A. (2007). O método de observação na psicologia: Considerações sobre a produção científica. Interação em Psicologia, 11(2), 199-210.

Carlson, J. S., Tiret, H. B., Bender, S. L., \& Benson, L. (2011). The influence of group training in the Incredible Years Teacher Classroom Management Program on preschool teachers' classroom management strategies. Journal of Applied School Psychology, 27(2), 134-154. doi: 10.1080/15377903.2011.565277

Cohen, L., Manion, L., \& Morrison, K. (2007). Research methods in education (6th ed.). New York: Routledge.

Downer, J. T., Booren, L. M., Lima, O. K., Luchner, A. E., \& Pianta, R. C. (2010). The Individualized Classroom Assessment Scoring System (inCLASS): Preliminary reliability and validity of a system for observing preschoolers' competence in classroom interactions. Early Childhood Research Quarterly, 25(1), 1-16. doi: 10.1016/j.ecresq.2009.08.004

Gaspar, M. F., Leitão, S., \& Vale, V. (2011). The Teacher-Pupil Observation Tool (T-POT): Manual de cotação. Manual não publicado. (Tradução e adaptação do Coding Manual de Pam Martin-Forbes, 2009).

Harms, T., Clifford, R. M., \& Cryer, D. (1998). Early Childhood Environment Rating Scale-Revised. New York: Teachers College.

Hutchings, J., Daley, D., Jones, K., Martin, P., Bywater, T., \& and Gwyn, R. (2007). Early results from developing and researching the Webster-Stratton Incredible Years Teacher Classroom Management Training Programme in North West Wales. Journal of Children's Services, 2(3), 15-26.

Hutchings, J., Martin-Forbes, P., Daley, D., \& Williams, M. E. (2013). A randomized controlled trial of the impact of a teacher classroom management program on the classroom behavior of children with and without behavior problems. Journal of School Psychology, 51(5), 571-585. doi: 10.1016/j.jsp.2013.08.001

Hyson, M., Hirsh-Pasek, K., \& Rescorla, L. (1990). The classroom practices inventory: An observational instrument based on the NAEYC's guidelines for developmentally appropriate practices for four and five-year-old children. Early Childhood Research Quarterly, 6, 183-197. doi: 10.1016/0885-2006(90)90015-S

Lewis, T. J., Scott, T. M., Wehby, J. H., \& Howard, P. (2014). Direct observation of teacher and student behavior in school settings: Trends, issues and future directions. Behavioral Disorders, 39(4), 190-200.

Martin, P. A., Daley, D., Hutchings, J., Jones, K., Eames, C., \& Whitaker, C. J. (2010). The Teacher-Pupil Observation Tool (T-POT): Development and testing of a new classroom observation measure. School Psychology International, 31(3), 229-249. doi: $10.1177 / 0143034310362040$

McGilloway, S., Hyland, L., Ní Mháille, G., Lodge, A., O’Neill, D., ... Donnelly, M. (2010). Positive classrooms, positive children: A randomized controlled trial to investigate the effectiveness of the Incredible Years Teacher Classroom Management programme in an Irish context (short-term outcomes). Dublin: Archways.

Merrell, K. W. (2008). Behavioral, social, and emotional assessment of children and adolescents (3rd ed.). New York: Erlbaum.

Pelham, W. E., Greiner, A. R., \& Gnagy, E. M. (2008). Student Behavior Teacher Response Observation Code Manual. Unpublished Manual.

Pianta, R. C., LaParo, K. M., \& Hamre, B. K. (2008). Classroom Assessment and Scoring System manual, Pre-K. Baltimore, MD: Paul H. Brookes.

Pianta, R. C., \& Stuhlman, M. W. (2004). Teacher-child relationships and children's success in the first year of school. School Psychology Review, 33(3), 444-458.

Robinson, E. A., \& Eyberg, S. (1981). The Dyadic Parent-Child Interaction Coding System: Standardization and validation. Journal of Consulting and Clinical Psychology, 49(2), 245-250. doi: 10.1037/0022-006X.49.2.245

Scott, T. M., Alter, P. J., \& Hirn, R. G. (2011). An examination of typical classroom context and instruction for students with and without behavioral disorders. Education and Treatment of Children, 34(4), 619-641. doi: 10.1353/etc.2011.0039

Strickland, J., Hopkins, J., \& Keenan, K. (2012). Mother-teacher agreement on preschoolers' symptoms of ODD and CD: Does context matter? Journal of Abnormal Child Psychology, 40(6), 933-943. doi: 10.1007/s10802-012-9622-y

Thangarajathi, S., \& Enok J. T. (2010). Classroom management: A challenging task for the teachers. Journal on Educational Psychology, 4(2), 11-18.

Volpe, R. J., DiPerna, J. C., Hintze, J. M., \& Shapiro, E. S. (2005). Observing students in classroom settings: A review of seven coding schemes. School Psychology Review, 34(4), 454-474. 
Vujnovic, R. K., Fabiano, G. A., Waschbusch, D. A., William E. Pelham, W. E., Greiner, A. ...Buck, M. (2014). Preliminary psychometric properties of an observation system to assess teachers' use of effective behavior support strategies in preschool classrooms. Education and Treatment of Children, 37(2), 323-346. doi: 10.1353/etc.2014.0020

Webster-Stratton, C. (2003). The Teacher Classroom Management Program (Rev. ed.). Washington, DC: Incredible Years Ltd.

Webster-Stratton, C., Reid, M. J., \& Stoolmiller, M. (2008). Preventing conduct problems and improving school readiness: Evaluation of the Incredible Years Teacher and Child training programs in high-risk schools. Journal of Child Psychology and Psychiatry, 49(5), 471-488. doi: 10.1111/j.1469-7610.2007.01861.x

\section{Sobre as autoras}

Sofia O. Major é psicóloga, doutora em Avaliação Psicológica pela Faculdade de Psicologia e de Ciências da Educação da Universidade de Coimbra e professora auxiliar na Faculdade de Ciências Sociais e Humanas da Universidade dos Açores.

Maria João Seabra-Santos é psicóloga, doutora em Avaliação Psicológica, professora auxiliar na Faculdade de Psicologia e de Ciências da Educação da Universidade de Coimbra e membro do Centro de Investigação em Neuropsicologia e Intervenção Cognitivo-Comportamental (CINEICC).

Maria Filomena Gaspar é psicóloga, doutora em Psicologia da Educação pela Faculdade de Psicologia e de Ciências da Educação da Universidade de Coimbra e professora associada na Faculdade de Psicologia e de Ciências da Educação da Universidade de Coimbra.

Mariana Pimentel é psicóloga e mestre em Psicologia pela Faculdade de Psicologia e de Ciências da Educação da Universidade de Coimbra.

Andreia F. Azevedo é psicóloga, doutora em Psicologia Clínica pela Faculdade de Psicologia e de Ciências da Educação da Universidade de Coimbra e mentora no Programa Básico para Pais Anos Incríveis.

Tatiana C. Homem é psicóloga clínica, doutora em Psicologia Clínica pela Faculdade de Psicologia e de Ciências da Educação da Universidade de Coimbra e mentora no Programa Básico para Pais Anos Incríveis.

Elsa Baptista é psicóloga, mestre em Psicologia pela Faculdade de Psicologia e de Ciências da Educação da Universidade de Coimbra e bolsista de Investigação no Hospital Pediátrico do Centro Hospitalar e Universitário de Coimbra. 